Questions vives

\section{Questions Vives}

Recherches en éducation

Vol. $6 n^{\circ} 12$ | 2009

Evaluer les enseignants et les formateurs. Comment? Pourquoi ? Pour quoi ?

\title{
La première inspection des professeurs stagiaires de l'enseignement agricole français
}

Julie Blanc et Jean-François Marcel

\section{(2) OpenEdition}

Journals

Édition électronique

URL : http://journals.openedition.org/questionsvives/420

DOI : $10.4000 /$ questionsvives.420

ISBN : 978-2-8218-1084-6

ISSN : $1775-433 \mathrm{X}$

Éditeur

Université Aix-Marseille (AMU)

Édition imprimée

Date de publication : 6 juin 2009

Pagination : 77-91

ISBN : 978-2-912643-36-0

ISSN : 1635-4079

\section{Référence électronique}

Julie Blanc et Jean-François Marcel, «La première inspection des professeurs stagiaires de l'enseignement agricole français », Questions Vives [En ligne], Vol. 6 n $^{\circ} 12$ | 2009, mis en ligne le 01 janvier 2011, consulté le 01 mai 2019. URL : http://journals.openedition.org/questionsvives/420 DOI : 10.4000/questionsvives.420

\section{(c) (i) (9)}

Questions Vives est mis à disposition selon les termes de la licence Creative Commons Attribution Pas d'Utilisation Commerciale - Pas de Modification 4.0 International. 


\title{
La première inspection des professeurs stagiaires de l'enseignement agricole français
}

\author{
Julie Blanc ${ }^{1}$, Jean-François Marcel$^{2}$
}

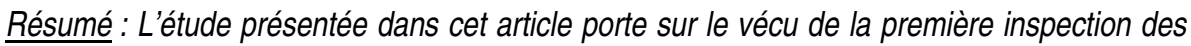
professeurs stagiaires de l'enseignement agricole. Partant du modèle théorique de la causalité triadique réciproque emprunté à Bandura (1980, 2003), les pratiques du stagiaire lors de l'inspection sont envisagées comme relevant d'interactions mutuelles entre son histoire professionnelle et personnelle, le contexte écologique du stage pédagogique et le déroulement de la séance inspectée. Le dispositif méthodologique repose sur un matériel discursif tiré d'entretiens semi-directifs menés auprès de huit professeurs stagiaires. Les résultats font apparaître trois types de vécus d'inspection: l'inspection vécue comme un moment de formation, comme un jugement technique ou encore comme un châtiment. Le contexte humain et matériel, l'expérience professionnelle du stagiaire ou encore le déroulement de la séance interviennent comme des facteurs de variation de vécus et influencent de façon différenciée l'identité professionnelle des futurs enseignants.

Mots-clés: Professeur stagiaire, inspection, vécu, identité professionnelle, enseignement agricole français.

Abstract: The study presented in this article concerns student teachers' personal experience of their first inspection, in the context of agricultural education. Using the conceptual model of triadic reciprocal causation borrowed from Bandura (1980, 2003), the inspected student teachers' practices are seen as an interactional causal structure made up of the student teachers' professional and personal history, the environment in which teaching practice is set and the course of the inspected session. The methodology is based on discourse extracts taken from the semi directive interviews of eight student teachers. The results bring to light three kinds of personal experience: inspection taken as a training period, as a test or as a punishment. The material and human context, the student teacher's professional background and the course of the teaching session appear as variation's factors of the way the student goes through the inspection. These factors influence the professional identity of the future teachers differently.

Keywords: Student teacher, inspection, personal experiences, professional identity, French agricultural education.

\footnotetext{
1 Post-Doctorante, Toulouse EducAgro, ENFA, Université de Toulouse.

2 Professeur, Toulouse EducAgro, ENFA, Université de Toulouse.
} 
Julie Blanc, Jean-François Marcel

La première inspection des professeurs stagiaires

\section{La première inspection des professeurs stagiaires de l'enseignement agricole}

\section{Introduction}

La thématique de l'évaluation des enseignants, sa raison d'être ainsi que les modalités de sa mise en œuvre, traitée dans ce numéro, met en lumière une composante fondamentale dans toute carrière professionnelle. L'évaluation des travailleurs, plus précisément des professionnels de l'éducation et de la formation, s'inscrit à la fois dans le champ du développement professionnel et dans le champ social. L'enseignant doit en effet « rendre des comptes ", même s'il est davantage soumis à une obligation de moyens mis en œuvre qu'à une obligation de résultats (Perrenoud, 1996). Cette double référence rend sa réalisation (pourtant nécessaire) et sa définition complexes car plusieurs types d'évaluations coexistent, différant selon les objectifs poursuivis et selon les logiques qui les sous-tendent. Nous traiterons ici de l'inspection comme contrôle des pratiques enseignantes.

La formation des professeurs du secondaire inclut, au Ministère de l'Agriculture, comme à l'Éducation Nationale un contrôle de l'exercice professionnel des futurs enseignants et donc une première inspection. II s'agit d'une évaluation qui d'une part, participera à l'examen de qualification professionnelle (EQP) en vue de valider et ponctuer l'ensemble d'une année de formation en permettant la titularisation; et qui d'autre part amorcera le début de leur carrière. En ce sens, l'inspection des professeurs stagiaires durant leur année de formation initiale constitue un enjeu essentiel en matière d'évaluation du travail des enseignants. L'importance que revêt toute inspection, et de façon exacerbée la première d'une carrière, mérite que l'on s'y attarde en interrogeant précisément le point de vue des professeurs stagiaires.

Cet article porte sur le vécu de la première inspection, l'objectif est de saisir le déroulement des séances inspectées au travers du point de vue des stagiaires ainsi que ses conséquences en termes d'identité et de développement professionnels.

Dans un premier temps, nous développerons l'hypothèse générale qui sous-tend ce travail de recherche ainsi que le cadre explicatif proposé. Nous caractériserons ensuite l'inspection comme type de pratiques d'évaluation à l'aune du cadre d'analyse avancé par Paquay, 2004. Nous indiquerons pour ce faire ses objets et référents, sa fonction (les buts et les enjeux de l'évaluation), les acteurs en présence (l'auteur et le destinataire de l'évaluation), le moment (le contexte de l'évaluation) ainsi que les moyens (les outils et dispositifs, le traitement et la communication de l'évaluation) (Paquay, 2004). Outre clarifier notre objet d'étude, cette caractérisation permettra de pointer les spécificités d'une inspection dans le cadre du système agricole. Après avoir précisé nos choix méthodologiques, nous présenterons puis discuterons les résultats. 


\section{Présentation de la recherche}

\subsection{Le vécu de l'inspection: cadre théorique}

La théorie sociocognitive de la causalité triadique réciproque (Bandura, 1980) nous permettant d'appréhender le comportement de l'enseignant comme le produit d'interactions dynamiques entre trois séries de facteurs (caractéristiques personnelles de l'enseignant, son action, le contexte) sera ici mobilisée comme cadre explicatif.

Pour expliquer le comportement humain, Bandura $(1980,2003)$ utilise la conception de l'interaction puisée dans la théorie de l'apprentissage social. Le terme de causalité renvoie à l'idée de dépendance fonctionnelle entre différents types de facteurs. Ainsi, "les facteurs personnels internes (événements cognitifs, émotionnels et biologiques), les comportements et l'environnement opèrent tous comme des facteurs en interaction qui s'influencent réciproquement »(Bandura, 2003). Cette réciprocité n'implique cependant pas que les trois groupes de facteurs aient le même impact.

Si nous transposons ce modèle théorique à notre objet d'étude, le stagiaire (son histoire personnelle et professionnelle) se situerait sur le pôle "caractéristiques personnelles ", la séance inspectée au sein de laquelle s'actualisent à la fois les pratiques de l'inspecté et celles de l'inspecteur, sur le pôle "action" et enfin le contexte, à savoir l'établissement d'accueil, la discipline enseignée etc. sur le pôle "environnement ». L'interaction mutuelle entre ces trois pôles nous permet ainsi d'appréhender les pratiques de l'enseignant stagiaire lors de la séance d'inspection. Remarquons toutefois que ces pratiques ne sont accessibles, dans la présente étude, qu'au travers de ce qu'en disent les acteurs, notre analyse se focalisera donc sur le vécu de l'inspection par l'enseignant stagiaire.

À partir de ce cadre théorique, nous formulons l'hypothèse de recherche suivante, il existe une diversité de vécus d'inspection selon:

-la séance inspectée et les pratiques d'inspection,

-la dimension écologique, au niveau micro (la classe, la discipline enseignée...) et au niveau meso (l'établissement d'accueil, l'équipe éducative, le conseiller pédagogique,...) ; -l'histoire professionnelle et personnelle du stagiaire.

\subsection{La première inspection : contexte de la recherche}

Les pratiques pédagogiques du professeur stagiaire dans l'enseignement agricole, nous l'avons vu, sont l'objet de l'évaluation dont il est question dans cet article. Sa fonction est de vérifier, de contrôler si le futur professeur remplit sa mission d'enseignement, et ainsi de le " déclarer apte " à la pratique du métier. L'évaluation constitue ainsi un processus de validation de compétences professionnelles. Dans le second degré (y compris dans le système agricole), elle est effectuée par des inspecteurs pédagogiques chargés donc d'évaluer les performances des stagiaires. Elle fait l'objet d'un rapport, remis à l'école de formation (ENFA ${ }^{3}$ ), dont le stagiaire n'est pas destinataire. Ce rapport est constitutif d'un dossier comportant en outre une évaluation du proviseur du lycée d'accueil, une évaluation du conseiller pédagogique dans la discipline ainsi qu'une évaluation des formateurs de l'ENFA. Ce dossier est ensuite examiné lors de la réunion du jury en vue de l'évaluation certificative. La première inspection se déroule le plus souvent à l'occasion du troisième

3 École Nationale de Formation Agronomique. 
stage en établissement d'accueil (stage dit de " pratique autonome »). Enfin, concernant les modalités, l'inspecteur assiste, dans un premier temps, à deux séances de cours et travaux pratiques. II s'agit d'une observation instrumentée à l'aide d'une grille (comprenant des indicateurs basés sur des critères définis par rapport aux attentes disciplinaires et institutionnelles) et d'un dossier spécifique. Dans un second temps, un entretien appelé aussi débriefing est proposé. L'inspecteur, à ce moment là, expose les points forts et les points faibles de la pratique du stagiaire en suivant le déroulé de la séance. L'objectif est également de mettre en évidence des points de didactique "non conformes aux attendus disciplinaires" et des "écarts par rapport aux attentes institutionnelles" ainsi que de proposer des perspectives d'évolutions attendues ${ }^{4}$. Le débriefing se clôt sur une discussion avec le conseiller pédagogique et le stagiaire.

\subsection{Choix méthodologiques}

Cet article se propose d'analyser, dans une visée heuristique, les situations d'inspection et leur vécu en privilégiant le point de vue des professeurs stagiaires. En d'autres termes, c'est au travers de leur discours que nous allons tenter d'accéder à une intelligibilité du vécu d'inspection. Pour ce faire, nous avons retenu une méthodologie de type qualitatif (Miles \& Huberman, 2003). Notre exposé accordera d'ailleurs, une large part aux verbalisations des futurs enseignants afin que la dimension du "vécu " prenne tout son sens.

L'échantillon se compose de huit stagiaires en formation à l'ENFA. Nous avons conduit une série d'entretiens semi-directifs à l'issue du stage en établissement dit de pratique autonome. Les questions de recherche visaient à recueillir une description et une analyse de la séance inspectée. Ainsi, nous avons interrogé les stagiaires sur la préparation (en amont de l'inspection), la réalisation (pendant) ainsi que sur l'entretien qui suit. Enfin, nous leur avons demandé de nous faire part de leur vécu, de leur ressenti à l'égard de cette première inspection.

Sur le plan du traitement des données, afin de découper puis de classer notre matériel discursif en vue d'une analyse de contenu, nous avons identifié des catégories extrinsèques à partir du modèle théorique de la causalité triadique réciproque ${ }^{5}$ (Bandura, 1980, 2003), qui constituera in fine le squelette de notre analyse. À partir de ces larges catégories de départ, nous avons procédé à une analyse de contenu thématique des verbatim (Bardin, 1993; Mucchielli, 1998) qui a consisté en un relevé et un classement de différents thèmes, patterns. Celle-ci nous a conduit à préciser et à compléter nos catégories à l'aide de variables (ex: Durant la préparation de la séance inspectée, le stagiaire a reçu l'aide de son conseiller pédagogique), en suivant la chronologie et les moments-clés du déroulement de l'inspection. Ces différentes étapes dans l'analyse ont permis d'aboutir à un descriptif exhaustif des séances, des préparations, des conditions d'accueil et des entretiens (débriefings) rapportés par chaque stagiaire.

\footnotetext{
${ }^{4}$ Éléments recueillis au cours d'échanges avec des inspecteurs de l'enseignement agricole.

${ }^{5}$ En regard de ce modèle théorique, nous nous devions de prendre en compte, outre les variables relatives à l'inspection, les variables relatives au contexte de stage en établissement d'accueil. Ceci a permis d'élargir le spectre de notre compréhension du vécu de l'inspection par les professeurs stagiaires.
} 
Evaluer les enseignants et les formateurs

Comment, pourquoi, pour quoi ?

\section{Résultats de l'enquête et discussion}

Une première lecture des données témoigne d'une diversité d'inspections vécues. En approfondissant l'analyse, ont émergé des régularités qui nous ont conduit à classer des familles de discours et par voie de conséquence à les discriminer.

Notre exposé sera structuré autour des trois composantes du modèle théorique déjà cité : le déroulement de la séance inspectée (action du stagiaire, action et attitude de l'inspecteur), le contexte (les conditions d'accueil dans l'établissement de stage...) enfin, les caractéristiques personnelles et professionnelles du stagiaire. Après avoir synthétisé à l'aide d'un tableau les éléments saillants des données, nous ferons état de deux angles d'approche : ce qui varie dans les discours des stagiaires puis ce qui, au contraire, permet de les regrouper.

\subsection{Catégories et variables retenues : variations au sein d'un système tripartite}

Le tableau 1 (page suivante) propose donc de décrire et de condenser les éléments d'analyse en fonction des catégories du système tripartite emprunté à la théorie sociocognitive de Bandura (2003) et découpées en variables.

\section{La séance inspectée et les pratiques d'inspection}

Des variations ont été relevées dans le discours des stagiaires notamment pour ce qui est du réalisé en amont de l'inspection (sa préparation). Certains stagiaires au contraire d'autres ont pu bénéficier d'un entraînement en testant leur séance avec un autre groupe d'élèves. Cette mise en situation semble favoriser une aisance et un meilleur déroulement de la séance au moment de l'inspection.

L'analyse met aussi en lumière des variations d'attitudes des inspecteurs. Ainsi, pour certains stagiaires l'attitude de l'inspecteur inhibe tout échange et justification des pratiques de la part des professeurs stagiaires. Par conséquent, si dans certains cas, l'inspecteur peut proposer une inspection orientée vers l'accompagnement, le conseil et se montrer rassurant vis-à-vis des stagiaires, dans d'autres à l'inverse, son approche sera davantage réprobatrice. Ces éléments sont à corréler de façon évidente avec le vécu global de l'inspection déclaré par les stagiaires. Le stagiaire déclare en effet avoir bien vécu sa première inspection lorsque l'inspecteur s'est montré compréhensif ou neutre. En revanche, l'attitude dépréciatrice de l'inspecteur induit un ressenti opposé. Ainsi, sur la question du vécu de la première inspection dans sa globalité, l'attitude de l'inspecteur constitue un premier « baromètre ».

\section{Le contexte humain et matériel}

Le contexte, envisagé dans son sens le plus large, à savoir humain et matériel constitue en outre un facteur de variation de vécus d'inspection. Certains professeurs stagiaires sont bien accueillis et se sentent appartenir à l'établissement d'accueil, d'autres sont sinon mis à l'écart tout au moins non considérés au sein de l'équipe pédagogique et éducative. Par ailleurs, pour certains le conseiller pédagogique apparaît comme une ressource et même un allié, tandis que pour d'autres, le relationnel avec celui-ci s'avère beaucoup plus distant. II ressort aussi que le conseiller pédagogique a pu intervenir directement dans la préparation de la séance en délivrant des conseils et autres méthodologies de travail pour certains stagiaires, tandis que pour d'autres son apport s'est révélé plus limité. 
Julie Blanc, Jean-François Marcel

La première inspection des professeurs stagiaires

\begin{tabular}{|c|c|c|c|c|}
\hline \multirow{2}{*}{\multicolumn{2}{|c|}{$\begin{array}{l}\text { Catégories } \\
\text { Variables }\end{array}$}} & \multirow{2}{*}{\multicolumn{3}{|c|}{ Eléments d'analyse tirés des entretiens }} \\
\hline & & & & \\
\hline \multirow{3}{*}{$\begin{array}{l}\text { Séance } \\
\text { inspectée } \\
\text { et pratiques } \\
\text { d'inspection }\end{array}$} & $\begin{array}{l}\text { Préparation } \\
\text { de la séance }\end{array}$ & $\begin{array}{l}\text { Le stagiaire a testé sa } \\
\text { séance }\end{array}$ & $\begin{array}{l}\text { Le stagiaire n'a pas } \\
\text { bénéficié } \\
\text { d'entraînement }\end{array}$ & \\
\hline & $\begin{array}{l}\text { Vécu de la } \\
\text { séance / de } \\
\text { l'inspection }\end{array}$ & $\begin{array}{l}\text { Bon ressenti du } \\
\text { stagiaire à l'égard de sa } \\
\text { prestation. } \\
\text { Vécu positif de } \\
\text { l'inspection }\end{array}$ & $\begin{array}{l}\text { Bon ressenti du } \\
\text { stagiaire à l'égard de sa } \\
\text { prestation. } \\
\text { Vécu négatif de } \\
\text { l'inspection }\end{array}$ & $\begin{array}{l}\text { Ressenti mitigé du } \\
\text { stagiaire à l'égard de } \\
\text { sa prestation. } \\
\text { Vécu positif de } \\
\text { l'inspection }\end{array}$ \\
\hline & $\begin{array}{l}\text { Attitude de } \\
\text { l'inspecteur }\end{array}$ & $\begin{array}{l}\text { Débriefing basé sur } \\
\text { l'échange. Opportunité } \\
\text { a été laissée au } \\
\text { stagiaire de se justifier, } \\
\text { d'expliciter ses } \\
\text { pratiques pédagogiques } \\
\text { L'inspecteur s'est voulu } \\
\text { rassurant et a délivré } \\
\text { de nombreux conseils } \\
\text { et ficelles du métier: } \\
\text { posture } \\
\text { d'accompagnateur }\end{array}$ & $\begin{array}{l}\text { Pas d'opportunité pour } \\
\text { le stagiaire } \\
\text { d'argumenter ses } \\
\text { pratiques } \\
\text { pédagogiques. } \\
\text { L'inspecteur a fait } \\
\text { montre davantage d'une } \\
\text { attitude réprobatrice et a } \\
\text { délivré un discours peu } \\
\text { constructif : posture de } \\
\text { procureur/détracteur }\end{array}$ & $\begin{array}{l}\text { L'inspecteur se pose } \\
\text { en juge évaluateur, } \\
\text { la neutralité domine : } \\
\text { posture de juge }\end{array}$ \\
\hline \multirow[b]{2}{*}{$\begin{array}{l}\text { Contexte } \\
\text { humain et } \\
\text { matériel }\end{array}$} & $\begin{array}{l}\text { Etablissement } \\
\text { d'accueil }\end{array}$ & $\begin{array}{l}\text { Bonne intégration, vécu } \\
\text { positif des stages en } \\
\text { établissement }\end{array}$ & $\begin{array}{l}\text { Difficulté d'intégration } \\
\text { dans l'équipe } \\
\text { pédagogique du lycée }\end{array}$ & \\
\hline & $\begin{array}{l}\text { Nature des } \\
\text { rapports } \\
\text { entretenus } \\
\text { avec le } \\
\text { conseiller } \\
\text { pédagogique }\end{array}$ & $\begin{array}{l}\text { Nombreux échanges, } \\
\text { interactions riches. } \\
\text { Aide soutenue à la } \\
\text { préparation de la } \\
\text { séance inspectée }\end{array}$ & $\begin{array}{l}\text { Relations caractérisées } \\
\text { par la distance. } \\
\text { Le conseiller } \\
\text { pédagogique n'incarne } \\
\text { pas une ressource } \\
\text { pédagogique pour le } \\
\text { stagiaire }\end{array}$ & \\
\hline \multirow{2}{*}{$\begin{array}{c}\text { Histoire } \\
\text { personnelle et } \\
\text { professionnelle } \\
\text { du stagiaire }\end{array}$} & $\begin{array}{l}\text { Expérience } \\
\text { professionnelle }\end{array}$ & $\begin{array}{l}\text { Expérience de } \\
\text { contractuel } \\
\text { Vocation pour le métier } \\
\text { d'enseignant }\end{array}$ & $\begin{array}{l}\text { Pas d'expérience } \\
\text { professionnelle } \\
\text { Pas de vocation a priori } \\
\text { pour le métier } \\
\text { d'enseignant }\end{array}$ & \\
\hline & $\begin{array}{l}\text { Identité } \\
\text { professionnelle }\end{array}$ & $\begin{array}{l}\text { Renforcement du } \\
\text { Sentiment d'Efficacité } \\
\text { Professionnelle. } \\
\text { Le stagiaire se sent } \\
\text { réellement et se dit être } \\
\text { enseignant une fois } \\
\text { l'inspection passée. }\end{array}$ & Régression identitaire & $\begin{array}{l}\text { Progression lente } \\
\text { vers une identité } \\
\text { enseignante. } \\
\text { L'inspection ne } \\
\text { constitue pas à elle } \\
\text { seule le point de } \\
\text { basculement d'une } \\
\text { identité de stagiaire } \\
\text { à une identité } \\
\text { d'enseignant. }\end{array}$ \\
\hline
\end{tabular}

Tab. 1 : synthèse des éléments d'analyse en fonction des catégories et des variables mises en évidence 
Evaluer les enseignants et les formateurs

Comment, pourquoi, pour quoi ?

\section{L'histoire personnelle et professionnelle du stagiaire}

Parmi les professeurs stagiaires que compte notre échantillon, certains ont bénéficié d'une expérience professionnelle en tant que contractuels. Leur expérience de terrain leur a probablement permis d'avoir le recul nécessaire vis-à-vis de leurs pratiques pédagogiques et d'envisager le discours de l'inspecteur de façon plus pragmatique qu'un stagiaire découvrant le métier.

Du point de vue de l'identité professionnelle, plusieurs discours apparaissent. Si pour certains stagiaires, la première inspection ne demeure qu'une étape dans la formation, pour d'autres, elle constitue un cap important, le point de basculement d'une identité de stagiaire à une identité d'enseignant, de professionnel. Dans le cas de vécus difficiles, elle déclenche une régression identitaire.

\subsection{Trois expériences de la première inspection}

Le tableau 1 met en exergue des variations dans les vécus d'inspection. II esquisse les trois familles de discours qui ressortent de notre analyse. Ces dernières nous ont conduits à distinguer trois types de vécus d'inspection : l'inspection vécue comme un moment de formation, vécue comme un jugement technique ou encore vécue comme un châtiment. L'exposé suivant qui s'attachera à les détailler, conservera la structure tripartite de notre modèle théorique.

\section{L'inspection « formation " \\ - La séance inspectée}

Ce type de vécu d'inspection concerne des professeurs qui ont un bon ressenti à l'égard de leur prestation et un vécu positif de l'inspection dans sa globalité : "ça s'est très bien passé " (S.6). Pour l'une des trois stagiaires classées dans cette configuration, de façon nette, les conseils et remarques de l'inspectrice ont directement été intégrés dans ses pratiques : "L'avantage, enfin ce que j'ai apprécié, c'est d'avoir mon inspection en tout début de stage donc ça a permis de discuter avec l'inspectrice ... pour moi ce que l'inspectrice va me dire, ça va me donner des lignes de conduite [...] et du coup c'est rassurant de pouvoir m'appuyer sur quelqu'un qui pour moi a la légitimité de me dire ça [...] je me suis servie de ses conseils par la suite, [...] les questions de fond et de contenus enseignés, j'essaie de les réinvestir de suite " (S.5). Dans le cas de cette stagiaire, l'inspection a rempli un rôle de formation notamment par le réinvestissement des divers conseils délivrés. Ces conseils lors du débriefing sont assimilés à des "lignes de conduite ", en quelques sortes "labellisées " puisque venant d'une autorité légitime.

L'inspecteur peut aussi intervenir directement au cours de la séance inspectée, assumant là encore un rôle formateur. Ainsi, dans le cas de S.2, l'inspecteur a fait des remarques sur le rythme (trop lent), au moment de la pause et a fait des suggestions à la stagiaire afin qu'elle oriente différemment la suite de la séance: « il est venu me voir à la pause et il m’a proposé de le restructurer différemment [...] donc après j'ai rebondi sur ce qu'il avait proposé, ça m'a un petit peu perturbée "; " je me suis dit, c'est certainement bénéfique pour moi si j'arrive à intégrer ce qu'il est en train de me proposer ".

Une autre forme du rôle formateur assumé par l'inspecteur a également été relevée lors de l'inspection d'une seconde stagiaire. Après l'avoir rassurée sur les intentions de son inspection, qu'il voulait davantage portée sur le conseil, il lui a demandé, lors de l'entretien, 
de formuler un retour réflexif à l'égard de sa prestation sur la séance : " qu'avez-vous pensé de votre séance ? ". II l'a en outre questionnée sur le document-élève qu'elle a proposé : «il m'a dit "comment vous pensez améliorer ce document ?" ". Ces deux situations témoignent d'une invitation à adopter une posture de "praticien réflexif " (Schön, 1993) sur ses pratiques et par conséquent, d'une perspective formative.

Nous venons de lister quelques caractéristiques relevant de la configuration que nous avons appelée inspection « formation ». L'analyse de discours permet de dégager un certain nombre de facteurs nous paraissant en lien avec ce type de vécu d'inspection, facteurs repérés chez les trois stagiaires concernés.

-Le contexte

D'un point de vue écologique, les stagiaires déclarent être bien intégrées dans l'établissement d'accueil ou au sein d'un petit groupe de collègues.

Elles ont toutes mentionné une aide soutenue de la part du conseiller pédagogique lors de la préparation de l'inspection, alimentée par une bonne entente et des interactions riches tout au long de chaque stage : " la CP nous a beaucoup appuyées, beaucoup aidées et ça s'est très bien passé " (S.6); " mon conseiller pédagogique s'est rendu complètement disponible" (S.2). Ces échanges avec le conseiller pédagogique concernant la séance d'inspection ont été intenses et ont de plus été très utiles à cette stagiaire : "j'ai beaucoup appris parce qu'entre quand je suis arrivée face à lui et que je lui ai proposé ce que j'allais faire et la version finale que j'ai proposée à l'inspecteur le jour $\mathrm{J}$, il y a eu une grosse évolution et ça je m'en resservirai parce que j'ai appris à structurer un document pour qu'il soit accessible à des BEPA, qui soit opérationnel, clair ». Cet extrait témoigne d'un travail accru en amont, en vue de préparer au mieux la séance d'inspection. Le conseiller pédagogique est ici un allié, une ressource pour le stagiaire qui apprend le métier.

Les trois stagiaires ont de plus bénéficié d'un entraînement, une sorte de mise en situation de la séance en vue de l'inspection, la veille (avec une autre classe, ou en demi groupe) ou à l'occasion du stage pratique précédent : " cette séquence on l'a testée la veille avec une classe de 1ère STAV [...] ce qui m’a permis de réajuster aussi la durée parce que je suis toujours trop lente" (S.2) ; " on a eu la chance de pouvoir faire une première fois le TP la veille avec un groupe et ça [...] je sais qu'autour de moi en biologie, ils ne l'ont pas tous fait et ceux qui l'ont fait, ont vraiment apprécié " (S.6). À travers ces éléments de discours, nous voyons à quel point une mise en situation, un test de la séance permet à l'enseignant apprenant d'ajuster ses pratiques, de s'entraîner sur autant de paramètres tels que le temps, la compréhension des élèves... et à quel point, cela peut également contribuer à une meilleure performance.

Sur le plan de l'inspection en elle-même, les entretiens post-séance se sont révélés enrichissants et rassurants pour les trois stagiaires. Pour S.5, l'inspection a eu lieu au tout début du troisième stage en établissement, ce qui a été perçu comme un avantage : "ça a permis de discuter avec l'inspectrice "; " c'était vraiment bien, c'était riche dans l'échange et c'était intéressant ". Elle a en effet, tiré profit des conseils de l'inspectrice et a réinvesti immédiatement dans la poursuite du stage, les remarques concernant notamment les contenus enseignés. Par conséquent, l'entretien, au travers des échanges avec l'inspecteur, lui a permis de disposer de "ressorts ", de ficelles du métier, pour la suite de son stage en pratique autonome. Elle caractérise d'ailleurs, ce face-à-face avec l'inspectrice de 
Evaluer les enseignants et les formateurs

Comment, pourquoi, pour quoi ?

"formateur ". L'aspect formation de l'inspection pour les autres stagiaires s'est incarné au travers de la position d'accompagnateur assumée par l'inspecteur, conférant à l'inspection et plus particulièrement au débriefing un aspect rassurant, réconfortant : "l'inspecteur nous a bien dit qu'il était là pour faire une inspection conseil, qu'il savait bien qu'on n'avait pas beaucoup enseigné cette année, que c'est le début etc. II m'a dit "ne vous inquiétez pas", à partir du moment où il avait dit ça, déjà ça démystifiait un peu la chose ». Nous voyons ici que l'inspection est aux antipodes de la représentation angoissante, perturbante voire " fantasmée " (Mosconi et Heideiger, 2003) que tout enseignant peut généralement avoir de celle-ci. Pour S.2, le débriefing était collectif, c'est-à-dire en présence des deux stagiaires inspectées et la stagiaire a trouvé cette approche intéressante : " c'était intéressant [...] moi j'ai trouvé ça bénéfique parce que ça dédramatise aussi un peu ce que peut dire l'inspecteur aussi bien pour elle que pour moi " (S.2).

Ces différents extraits montrent que les débriefings avec l'inspecteur ont été basés sur des échanges, au travers desquels les stagiaires ont pu exposer leur point de vue, argumenter leurs propres pratiques.

- L'histoire professionnelle et personnelle des stagiaires

L'inspection au cours de l'année de formation serait à envisager comme un moment-clé, pour les trois stagiaires. En effet, la parole de l'inspecteur est venue valider, conforter les pratiques permettant ainsi de renforcer leur sentiment d'efficacité professionnelle ${ }^{6}$ (Marcel, 2009 ; Marcel \& Chassagne, 2008) et d'asseoir une identité professionnelle de professeur : " il m'a dit, c'est bon, vous ne vous êtes pas trompée de métier donc c'est un soulagement " (S.6) ; " j'ai considéré que c'était une reconnaissance de compétences que j'ai développées depuis plusieurs années " (S.2). Pour S.6, l'inspection s'est révélée être un temps fort, un tournant en termes d'identité, y compris dans le rapport aux autres enseignants de l'établissement. Elle a en effet remarqué un changement de comportement des autres professeurs vis-à-vis d'elle une fois l'inspection passée. L'inspection correspondrait ici à une sorte de rite initiatique donnant accès à la communauté qu'est le corps enseignant.

Enfin, notons que deux stagiaires ont une expérience professionnelle de contractuelle qui leur a probablement permis, grâce à leur recul, d'envisager l'inspection sous un angle de formation.

\section{L'inspection « jugement technique "}

-La séance inspectée

Cette configuration concerne également des stagiaires qui ont un vécu positif de l'inspection: " l'inspection s'est très bien passée, il n'y a pas eu de souci " (S.8) ; " une fois qu'on est dans le cours, tout se passe bien " (S.1). Ils déclarent avoir eu un bon vécu de séance et un bon sentiment à l'égard de leur prestation.

Au travers des discours des trois stagiaires concernés, nous relevons une certaine neutralité de l'inspecteur dont le compte-rendu au cours de l'entretien consiste à pointer les éléments positifs et les éléments négatifs. L'inspection prend alors la forme d'une évaluation normée et prescriptive : «il m'a dit qu'il fallait vraiment interroger tous les élèves même les

\footnotetext{
6 Dans ses travaux, Marcel $(2008,2009)$ adapte la notion de Sentiment d'Efficacité Personnelle empruntée à Bandura (2003) à son champ d'investigation qu'est la sphère professionnelle des enseignants, la notion se transforme alors en Sentiment d'Efficacité Professionnelle.
} 
plus discrets » (S.8) ; " il m'a dit que j'étais très rigoureuse et exigeante mais qu'il fallait que je fasse attention d'utiliser que le vocabulaire scientifique nécessaire " (S.1). L'inspecteur ne se veut, semble-t-il, volontairement pas "rassurant " comme dans le cas de figure précédent; la neutralité domine, il est ici juge, évaluateur: "je me faisais inspecter une heure le matin et une heure l'après midi, entre midi et deux je n'avais aucun signe de l'inspecteur c'est à dire je ne savais pas si ça s'était plutôt bien passé, plutôt moyen, plutôt mal [...] pas un sourire, rien dans le regard, moi ça m'a un petit peu préoccupée " (S.1).

Pour les trois stagiaires, nous observons une certaine concordance entre l'évaluation de l'inspecteur et l'autoévaluation du stagiaire : « je savais que c'était trop long, je le savais dès le départ [...] je m'étais dit, vu que c'était des histoires de gestion de temps, [...] je pensais qu'elle allait plus m'en tenir rigueur et en fait pas trop, elle-même m'a dit "c'était peut-être un petit peu ambitieux comme TP en 50 min" " (S.4) ; "tout était pertinent, il n'y a pas de problème, j'ai acquiescé tout ce qu'il a dit, tout était juste » (S.8).

Dans les trois cas, le debriefing s'est déroulé sous le mode de l'échange: "j'ai eu l'occasion de donner des justifications sur certaines choses" (S.4). Le stagiaire verbalise, justifie tandis que l'inspecteur interroge, conseille.

\section{- Le contexte}

Les différents points susmentionnés sont à mettre en lien avec des facteurs contextuels tels qu'une bonne intégration au sein de l'établissement de stage notamment pour deux stagiaires : " on est accueillis et acceptés comme de vrais titulaires " (S.8). Ce qui n'est en revanche pas le cas pour S.4 qui n'est pas parvenue à trouver sa place au sein de l'équipe pédagogique, souffrant probablement d'une image injustement négative du statut de stagiaire : " on n'a pas encore de vrais rapports d'égal à égal ».

Pour ce qui est de la préparation en amont de l'inspection, quelques points divergent. Seul un stagiaire a pu profiter d'un entraînement en testant la séance avec une autre classe. II a aussi pu bénéficier d'une aide de la part de son conseiller pédagogique tant sur le plan du contenu pédagogique de la séance que sur le plan organisationnel : " dans tout ce qui était la mise en œuvre à côté, la préparation des élèves, leur dire de bien arriver à l'heure, d'avoir leur vêtement de sécurité [...] il les a briefés pour qu'ils arrivent un peu avant, qu'ils aient bien leurs cahiers, donc il m'a bien aidé ". En revanche, pour S.4, la conseillère pédagogique n'a pas été une réelle ressource: "nos cours d'inspection, [...] elle les a regardés, vraiment regardés en détail le mercredi après-midi sachant que notre inspection était le jeudi matin, [...] on a peut-être changé deux trois petits trucs mais c'était plus le moment de tout changer ". Notons que les rapports entretenus avec la conseillère pédagogique sur l'ensemble des stages ont été peu favorables aux échanges: " on ne peut pas vraiment dire qu'on communique énormément » (S.4).

-L'histoire professionnelle et personnelle des stagiaires

L'impact de l'inspection sur l'identité professionnelle est ici moins manifeste, à l'exception d'une stagiaire pour qui l'avis de l'inspecteur a été prépondérant pour l'aider à se sentir désormais enseignante. Pour les autres, nous remarquons que si à l'issue du stage de pratique autonome, ils se sentent de plus en plus enseignants : "de plus en plus parce que ça s'est bien passé à l'inspection, là les jurys aussi se sont bien passés donc on bascule de plus en plus vers enseignant titulaire " (S.8), il semble néanmoins que la perspective de la rentrée " officielle ", en tant qu'enseignant véritablement responsable de classes, soit aussi 
Evaluer les enseignants et les formateurs

Comment, pourquoi, pour quoi ?

décisive : " je me sens encore un petit peu stagiaire, je pense, mais même à la rentrée je pense qu'il y aura toujours euh... [...] mais le fait d'assumer ses cours toute seule, de ne plus avoir quelqu'un, je vais forcément me sentir enseignante et plus stagiaire, ça sera fini" (S.4) ; " là je serai encore stagiaire quand même jusqu'au bout [...] au mois de septembre, ça sera différent je pense»(S.8). Ainsi, l'inspection joue un rôle important pour conforter l'enseignant stagiaire dans ses pratiques, en revanche, il semble qu'il faille attendre la fin de l'année de formation pour se départir complètement de «l'étiquette " stagiaire.

\section{L'inspection " châtiment "}

- La séance inspectée

Cette configuration concerne des professeurs stagiaires qui ont un vécu de l'inspection globalement négatif : « la première inspection ${ }^{7}$ [...] ça a été la déception du début à la fin [...] je me suis fait démolir [...], je n'ai pas eu un point positif, [...] après il y en a eu dans le rapport mais oralement j'en n'ai pas eu » (S.3) ; " l'entretien avec l'inspectrice ne s'est pas bien passé du tout... "; " pas du tout constructif... en sortant j'étais très déçue » (S.7). Le vocable "déception " est très présent dans le discours des deux stagiaires, notamment parce qu'elles n'ont pas obtenu les conseils attendus : "j'étais très déçue parce qu'en plus cette inspectrice, on l'avait rencontrée à l'ENFA, [...] et elle nous avait paru très humaine, dans l'échange, [...] et donc moi j'ai été à l'inspection en me disant, je vais avoir des conseils sur mes pratiques, et en fait ça n'a pas du tout été ça " (S.7) ; "ça a été la déception du début à la fin surtout qu'on l'avait vue avant, elle nous avait bien expliqué que c'était une visite plus conseil qu'autre chose, [...] mais ça ne s'est vraiment pas passé comme elle l'avait prédit » (S.3).

Cette configuration de vécu d'inspection se caractérise également par un hiatus entre le ressenti du déroulement de la séance et l'évaluation dont l'inspecteur fait part: " Moi je suis sortie de mes deux séances, pas satisfaite mais contente de ce que j'avais fait quand même [...] je doutais pas en sortant de la séance donc ça a été un choc, je ne m'y attendais pas " (S.3). Pour la seconde stagiaire : "la séance s'est bien déroulée "; "j'ai trouvé ça bien " à l'inverse, lorsqu'elle relate sa première inspection, son appréciation est sans ambiguïté : " j'ai raté mon inspection »; " je n'ai pas plu à l'inspectrice » (S.7).

Pour S.3, ce vécu difficile de l'inspection s'accompagne d'un sentiment d'injustice notamment par rapport à la prestation de sa collègue stagiaire 8 : "je pense qu'on a été dans les mêmes situations, on a fait à peu près les mêmes choses ". Manifestement, la stagiaire ne dispose pas de clés suffisamment explicites pour comprendre l'échec de cette première inspection même si, durant l'entretien, l'inspectrice a exposé les difficultés relevées.

Dans le cas de $S .8$, le sentiment d'injustice se détecte en filigrane: "elle a été très exigeante "; " elle venait chercher un prof de BTS ». Nous pouvons nous interroger en effet sur les raisons de ces exigences par rapport à la pratique avec des BTS alors que les séances inspectées ont concerné des secondes et des terminales. Ici, l'inspection est proche du contrôle : à savoir une confrontation des attentes préalables de l'inspecteur et de ce qu'il observe effectivement de la pratique de l'enseignant.

\footnotetext{
${ }^{7}$ Les deux stagiaires classées dans cette configuration ont subi une deuxième inspection.

8 Les stages en établissement sont effectués en binôme.
} 
Pour cette stagiaire aussi, l'incompréhension face à l'échec de la première inspection domine, d'autant plus qu'elle avait soumis sa préparation de séance à plusieurs référents : "j'avais montré mon TP à tous les enseignants du lycée, et à mon formateur de l'ENFA, donc j'avais été validée par l'ensemble de mes référents " (S.8).

Les occasions de se justifier ou d'argumenter leurs choix pédagogiques et leurs pratiques au cours de la séance ont été, pour les deux stagiaires, réduites à néant : " quand j'essayais de répondre avec l'argumentaire que j'avais à lui donner elle continuait sur ses points de vue " (S.3); "je n'ai pas parlé en fait, quand j'essayais de parler, elle me coupait la parole "; " j'étais d'accord avec certaines choses qu'elle disait mais c'était la façon de les dire et le fait de ne pas me laisser me défendre ça c'était très dur »(S.7).

- Le contexte

Ce type de vécu d'inspection va trouver en amont un certain nombre de facteurs que nous qualifierions de "non facilitants ", liés essentiellement au contexte de stage. Tout d'abord, une intégration difficile et problématique au sein de l'établissement où l'ambiance peut être qualifiée de peu agréable : " on ne se sent pas intégrées " (S.3).

En amont de la séance inspectée, S. 3 n'a pas trop pu compter sur l'aide de sa conseillère pédagogique avec qui elle entretient des rapports relativement distants : "elle ne s'intéresse pas plus que ça à ce qu'on fait " a-t-elle mentionné, comme si elle semblait lui reprocher son manque de disponibilité. Ainsi, la stagiaire déclare avoir préparé seule son inspection : " j’ai préparé seule, la CP n'a rien fait, moi j'aurais été seule dans un autre établissement, j'aurais fait la même chose, elle ne m'a rien apporté ". À l'inverse, S.7 parle d'une bonne entente avec son conseiller pédagogique, mais elle évoque une aide centrée " surtout sur la forme des documents" et ajoute qu'elle a bénéficié de l'aide du formateur en biologie: " mon formateur en bio c'était plus sur le fond, sur la réflexion ". Remarquons d'ailleurs que le formateur de biologie est aussi intervenu pour S.3 dans un second temps (pour les deux inspections) afin de valider en quelques sortes sa préparation de séance.

Enfin, les stagiaires n'ont pas eu l'occasion de répéter leur séance avant l'inspection, à l'exception de $\mathrm{S} .7$ pour la deuxième.

- L'histoire professionnelle et personnelle des stagiaires

L'analyse fait état de plusieurs points communs entre ces deux stagiaires. Elles avaient, nous l'avons vu, les mêmes attentes vis-à-vis de l'inspection et pensaient recueillir des conseils pour progresser dans leur pratique de novice. Notons en outre, qu'elles n'avaient, toutes deux, pas de vocation a priori pour le métier d'enseignant, leur projet professionnel étant d'abord orienté vers le cursus vétérinaire. Enfin, nous pouvons déceler de par leur expérience d'inspection difficile, une régression en termes identitaire. Pour S.3 en particulier, le redoublement prononcé entaille une identité professionnelle qui évoluait au cours des différents stages pédagogiques de plus en plus vers le statut de professeur. Pour S.7 qui a été inspectée une deuxième fois et qui valide tout de même l'année de formation, sans surprise, le point de vue se distingue légèrement. La deuxième inspection a été effectivement plus rassurante que la première qui l'avait rendu sceptique quant à sa capacité même à exercer le métier : " Je suis contente d'avoir été réinspectée [...] parce que ça m'a beaucoup déstabilisée [...], là je sais que je suis capable de passer une bonne inspection, ça a été vraiment important pour moi » (S.7). Ainsi, la deuxième inspection a été déterminante en 
Evaluer les enseignants et les formateurs

Comment, pourquoi, pour quoi ?

termes d'identité professionnelle, notamment parce qu'elle a été considérée comme une « collègue " par l'inspecteur.

\subsection{Synthèse et discussion}

Les résultats, nous l'avons vu, font ressortir une pluralité d'expériences de première inspection que nous avons classées selon trois configurations: inspections vécues comme un moment de formation, comme un jugement technique ou encore vécues comme un châtiment.

Ainsi, l'inspection est vécue différemment selon plusieurs critères: les pratiques et l'attitude de l'inspecteur, les conditions de préparation à l'inspection, le contexte d'accueil dans l'établissement, les relations entretenues avec le conseiller pédagogique, son degré d'implication dans la préparation de la séance inspectée et enfin, les caractéristiques personnelles du stagiaire, notamment son expérience professionnelle. Nous pouvons avancer concernant l'inspection "formation " que ces critères se sont avérés "facilitants " au bon déroulement de la séance inspectée, tandis que pour l'inspection " châtiment ", plutôt «non-facilitants ».

II apparaît en outre que l'ensemble de ces critères influence de façon différenciée l'identité professionnelle des futurs enseignants interrogés. Pour les stagiaires relevant du type d'inspection vécue comme un moment de formation, l'inspection, en renforçant leur sentiment d'efficacité professionnelle, a contribué à leur faire assumer un statut d'enseignant à part entière, une fois l'inspection passée. Pour d'autres, l'inspection n'intervient pas de façon directe et simultanée dans ce changement d'identité. Si elle contribue à valider les pratiques des stagiaires en quête de " reconnaissance " professionnelle voire à les rassurer, cette première inspection opère plus de façon diachronique. Les stagiaires déclarent en effet, se sentir vraiment enseignant qu'au moment où ils assumeront totalement la responsabilité d'une classe, à la rentrée scolaire de leur première année d'exercice. Enfin, pour d'autres, l'inspection s'est révélée déstabilisante, au point de remettre en question et de rétrograder une identité professionnelle enseignante certes balbutiante, à une identité de personnel encore en formation.

Ainsi, les vécus divergent en fonction de nombreux critères, nous pouvons donc inférer que cet état de fait a des conséquences en termes de développement professionnel en début de carrière. La première inspection, loin d'être une simple étape dans un processus de formation, intervient comme un catalyseur sur le développement professionnel du futur enseignant, la construction des savoirs d'une part et l'identité d'autre part. En effet, la première inspection a été l'occasion pour certains stagiaires de réinvestir des savoirs vicariants, des ficelles du métier construits dans l'échange avec l'inspecteur, pour d'autres en revanche, cette expérience a quelque peu inhibé leurs pratiques a posteriori et remis en question pour partie leur progression vers la titularisation.

Le paragraphe précédent montre que nous pouvons conforter notre hypothèse. Toutefois, la dimension écologique a été partiellement étudiée, seul le niveau meso (l'établissement d'accueil, les relations socioprofessionnelles avec l'équipe éducative ou le conseiller pédagogique) a pu être approfondi. Peu ou prou évoqué par les stagiaires interrogés, l'impact de la variable groupe-classe est difficile à appréhender dans le cadre précis d'une inspection, dans la mesure où généralement elle a lieu sur une séance de cours. II en va de même pour la variable discipline enseignée, sur laquelle les sujets interrogés ne peuvent se prononcer. 
Par conséquent, s'il s'avère pertinent de circonscrire une étude sur le vécu de la première inspection au discours des intéressés; rendre compte plus largement de pratiques d'inspection nécessite de s'orienter vers une méthodologie combinant situations observées et discours des deux types d'acteurs en présence. La confrontation " pratiques d'inspection " et " pratiques enseignantes » permettrait de prolonger la réflexion sur les interactions mutuelles entre les trois ensembles de facteurs étudiés et ainsi de saisir avec plus d'acuité les raisons de variations de situations d'inspection.

\section{En guise de conclusion}

Étudier les expériences d'inspection relatées par les stagiaires de l'échantillon nous a donné accès à leurs représentations, leur vécu. Cependant, cette pluralité de vécus peut faire écho à une pluralité de pratiques d'inspection et interroge de facto leur standardisation. L'objectif assigné à cette pratique d'évaluation en début de carrière est bien de valider une année de formation, déclarer un enseignant en apprentissage " apte " à enseigner, or le discours des stagiaires fait état de pratiques polymorphes fortement dépendantes elles aussi de divers éléments de contexte. Les différents vécus d'inspection mis ici en exergue constitueraient-ils des indices de distorsions évaluatives ${ }^{9}$ ? II semblerait en effet, qu'au-delà de la singularité de l'action, les pratiques, les façons de mener l'inspection ainsi que les exigences envers les formés divergent d'un inspecteur à l'autre et même d'une séance d'inspection à l'autre. Même s'il semble aller de soi que chaque individu, de par sa singularité, vive et ressente différemment diverses situations données, ceci pose davantage question dans le cas particulier de l'inspection, évaluation officielle du corps enseignant, censée être a minima standardisée, harmonisée pour garantir validité et objectivité.

Par conséquent, si la réponse à la question " pourquoi » évaluer les futurs enseignants paraît évidente dans la mesure où la titularisation et l'amorce de la carrière en sont les enjeux; cette étude montre que la question du "comment " reste à creuser afin de faciliter une entrée dans le métier (le " pour quoi ") garante d'un développement professionnel et d'une qualité d'enseignement optimisés.

\section{Bibliographie}

Bandura, A. (1980). L'apprentissage social. Bruxelles : Mardaga.

Bandura, A. (2003). Auto-Efficacité. Le Sentiment d'Efficacité Personnelle. Bruxelles: De Boeck.

Bardin, L. (1993). L'analyse de contenu. Paris : PUF.

Blanc, J. (2007). Construction et mobilisation de savoirs professionnels. Le cas des pratiques enseignantes d'évaluation à l'école primaire. Thèse de doctorat, Codirection: Marc Bru et Jean-François Marcel (non éditée).

Marcel, J.-F \& Chassagne, J.-M. (2008). Auto-évaluation et développement professionnel. Le cas des stagiaires de l'enseignement agricole français en fin de formation initiale. Actes du 20 ème colloque de l'ADMEE-Europe, Université de Genève.

Marcel, J.-F. (2009). Le Sentiment d'Efficacité Professionnelle, un indicateur pour connaître le développement professionnel des "nouveaux" professeurs de l'enseignement agricole français, Questions Vives, 5(11), 161-176.

${ }^{9}$ Expression empruntée à J.-M. Monteil (1999). 


\section{Evaluer les enseignants et les formateurs}

Comment, pourquoi, pour quoi ?

Miles, M.B. \& Huberman, M. (2003). Analyse des données qualitatives. Bruxelles : De Boeck. Monteil, J.-M. (1999). Proposition pour une nouvelle approche de l'évaluation des enseignants. Rapport remis au Ministre de l'Education Nationale, Claude Allègre. Ministère de l'éducation nationale, de la recherche et de la technologie.

Mucchielli, R. (1998). L'analyse de contenu des documents et des communications. Paris : ESF.

Paquay, L. (2004). (Ed.). L'évaluation des enseignants: tension et enjeux. Paris : l'Harmattan.

Perrenoud, P. (1996). L'évaluation des enseignants: entre une impossible obligation de résultats et une stérile obligation de procédure. L'Éducateur, 10, 24-30.

Schön, D. (1993). Le praticien réflexif. À la recherche du savoir caché dans l'agir professionnel. Montréal : Logiques. 